\title{
Editorial
}

\section{Clinical Methodology Matters in Epidemiology: Not All Benzodiazepines Are the Same}

\author{
Fiammetta Cosci ${ }^{a}$ Jenny Guidi ${ }^{b}$ Richard Balon ${ }^{c, d}$ Giovanni A. Fava ${ }^{b, e}$ \\ a Psychology and Psychiatry Unit, Department of Health Sciences, University of Florence, Florence, and ${ }^{\mathrm{b}}$ Department \\ of Psychology, University of Bologna, Bologna, Italy; Departments of ${ }^{\mathrm{C}}$ Psychiatry and Behavioral Neurosciences \\ and ${ }^{d}$ Anesthesiology, Wayne State University, Detroit, Mich., and ${ }^{e}$ Department of Psychiatry, State University of \\ New York at Buffalo, Buffalo, N.Y., USA
}

In 1977, at a time when psychiatric epidemiology was not as fashionable as it is today, Whitlock [1] outlined its aims and limitations. He criticized a narrow view of psychiatric epidemiology as concerned with the incidence, prevalence and distribution of mental disorders in the general population. He found 'the pursuit of investigations of this kind a singularly sterile activity, largely because it is difficult to see what possible purpose such enterprises have [...]. Head counting to establish prevalence norms is tedious and can rarely be carried out in any comprehensive fashion by those trained to recognize and treat mental disorders' [1, p. 11]. Studies concerned with prevalence rates, however, achieved a prominent role in the subsequent decades [2]. Even though such studies were not driven by financial conflicts of interest, they served an important commercial purpose. Expanding the target of psychotropic drug prescription to potentially universal consumption is facilitated by showing that psychiatric illness is widespread, undetected and undertreated [3]. Epidemiological studies are generally conducted using lay or poorly trained interviewers who cannot translate obtained data into clinical context and do not have the skills to judge whether the symptoms they elicit are severe enough to cause clinically significant distress or impairment [3]. There are very few exceptions to this rule [4]. (c) 2015 S. Karger AG, Basel

0033-3190/15/0845-0262\$39.50/0
Whitlock [1] emphasized the importance of specifying and testing explicit hypotheses in clinical epidemiology, particularly if such hypotheses might lead to practical measures to prevent and treat mental disorders. Pharmacoepidemiology, with particular reference to benzodiazepines, may illustrate the value of Whitlock's considerations.

For a long time benzodiazepines have provided an effective treatment for anxiety disorders [5], sleep disorders and a variety of medical conditions such as epilepsy and alcohol withdrawal [6]. Their large number of prescriptions raised many concerns and attempts at limiting their use [5]. The introduction of second-generation antidepressant and antipsychotic drugs has provided more expensive modalities of addressing anxiety disorders [7]. Substituting benzodiazepines with selective serotonin reuptake inhibitors (SSRI) and serotonin-noradrenaline reuptake inhibitors (SNRI) clearly appeared the commercial way to go. Such a road would have been difficult when new medications had to be compared to a gold standard, since direct comparisons clearly indicated higher efficacy and tolerability of benzodiazepines over antidepressants [8]. However, when such superiority was no longer required by regulatory agencies, alternative routes appeared. One was to perform comparisons by meta-analytic methods that are liable to manipulation instead of head-to-

\section{KARGER 125}

E-Mail karger@karger.com www.karger.com/pps 
head comparisons [9]. The other complementary strategy was to magnify the side effects of benzodiazepines. Since these negative aspects are actually very limited [5-8], the potential for dependency, toxicity and abuse had to be pictured dramatically, despite the fact that the percentage of recreational abuse is uncommon and the abuse is really low in relation to the number of people using them [10]. The reinforcing effects of benzodiazepines vary and are considerably weaker than those of other drugs of abuse such as other sedative hypnotics, stimulants and opiates [10]. On the other hand, the reinforcing effects are stronger that those of drugs recognized as having little abuse potential such as chlorpromazine [10].

In addition, similar [11], if not worse [12, 13], problems of dependence may ensue with SSRI and SNRI. The ghost of Alzheimer's disease was evoked in observational studies [14] despite a lack of supporting evidence [15]. Correlational methods in these investigations, however, appear to entail the risk of yielding spurious results when using highly heterogeneous constructs and populations. A very instructive example is provided by the alleged benefits of hormone replacement therapy in postmenopausal women as to coronary artery disease, which were reported by observational studies and contradicted by randomized controlled trials [16].

Clinical methodology matters in pharmacoepidemiology, and the study by Cloos et al. [17] provides an illustration of this point. Two distinct yet ostensibly related strategies were used. First, instead of grouping all benzodiazepines together, as is commonly done $[14,18]$, each medication was considered on its own. Second, the study tested a specific hypothesis formulated by Chouinard [19] in 2004, as suggested by Ciraulo et al. [20] and Schmauss et al. [21], namely, the presence of major clini- cal differences among benzodiazepines based on the joint consideration of relative lipid solubility, binding affinity and half-life. Drugs like triazolam and alprazolam, which have high lipid solubility, would be associated with higher dependence liability, cognitive impairment and anterograde amnestic effects [19]. On the contrary, benzodiazepines with low affinity for the benzodiazepine receptor and lipid solubility, such as clonazepam, would be associated with less dependence liability and amnestic potential [19].

In the 12-year national registry study performed in Luxembourg, about 4 out of 5 people who received a benzodiazepine were short-term or intermittent users [17]. Continuous use, not necessarily associated with dose escalation, occurred in the remaining cases. The initial pharmacological hypothesis was confirmed: alprazolam and triazolam were related to continuous and high-dose use, whereas clonazepam and clobazam were not [17]. Thus, it seems obvious that not all benzodiazepines are the same. The findings of the study by Cloos et al. [17] support the importance of a specific benzodiazepine selection based on a number of pharmacological aspects $[22,23]$. Unlike undifferentiated studies that provide little information to the clinician [18], the results indicate that certain benzodiazepines such as alprazolam and triazolam that are widely prescribed should be carefully used or simply avoided.

The type of pharmacoepidemiology portrayed in the study by Cloos et al. [17] provides a link between largescale and anecdotal clinical observations related to the side effects of medications [22,23]. It also calls for a more careful appraisal of the subtle psychological effects of various medications, including behavioral toxicity and iatrogenic comorbidity [24].

\section{References}

1 Whitlock FA: Psychiatric epidemiology: its uses and limitations. Aust NZ J Psychiatry 1977;11:9-18.

2 Kessler RC, Demler O, Frank RG, Olfson M, Pincus HA, Walters EE, Wang P, Wells KB, Zaslavsky AM: Prevalence and treatment of mental disorders, 1990-2003. N Engl J Med 2005;352:2515-2523.

3 Batstra L, Frances A: Holding the line against diagnostic inflation in psychiatry. Psychother Psychosom 2012;81:5-10.
4 Faravelli C, Abrardi L, Bartolozzi D, Cecchi C Cosci F, D’Adamo D, Lo Iacono B, Ravaldi C, Scarpato MA, Truglia E, Rosi S: The Sesto Fiorentino study: background, methods and preliminary results. Lifetime prevalence of psychiatric disorders in an Italian community sample using clinical interviewers. Psychother Psychosom 2004;73:216-225.

5 López-Muñoz F, Alamo C, García-García P: The discovery of chlordiazepoxide and the clinical introduction of benzodiazepines: half a century of anxiolytic drugs. J Anxiety Disord 2011;25:554-562.
6 Lasagna L: The role of benzodiazepines in nonpsychiatric medical practice. Am J Psychiatry 1977;134:656-658.

7 Balon R, Fava GA, Rickels K: Need for a realistic appraisal of benzodiazepines. World Psychiatry 2015;14:243-244.

8 Offidani E, Guidi J, Tomba E, Fava GA: Efficacy and tolerability of benzodiazepines versus antidepressants in anxiety disorders: a systematic review and meta-analysis. Psychother Psychosom 2013;82:355-362. 
9 Fava GA: Statistical alchemy for drug treatment of generalized anxiety disorder: a commentary on the meta-analysis by Baldwin et al. [BMJ 2011;342:d1199]. Psychother Psychosom 2011;80:261-263.

10 Task Force Report of the American Psychiatric Association: Benzodiazepine Dependence, Toxicity, and Abuse. Washington, American Psychiatric Association, 1990.

11 Nielsen M, Hansen EH, Goetzsche PC: What is the difference between dependence and withdrawal reactions? A comparison of benzodiazepines and selective serotonin re-uptake inhibitors. Addiction 2011;107:900-908.

12 Fava GA, Gatti A, Belaise C, Guidi J, Offidani E: Withdrawal symptoms after selective serotonin reuptake inhibitor discontinuation: a systematic review. Psychother Psychosom 2015;84:72-81.

13 Chouinard G, Chouinard VA: New classification of selective serotonin reuptake inhibitor withdrawal. Psychother Psychosom 2015;84: 63-71.
14 Billioti de Gage S, Moride Y, Ducruet T, Kurth T, Verdoux H, Tournier M, Pariente A, Bégaud $\mathrm{B}$ : Benzodiazepine use and risk of Alzheimer's disease: case-control study. BMJ 2014;349:g5205.

15 Salzman C, Shader RI: Benzodiazepine use and risk for Alzheimer disease. J Clin Psychopharmacol 2015;35:1-3.

16 Lawlor DA, Davey Smith G, Ebrahim S: Commentary: the hormone replacement-coronary heart disease conundrum: is this the death of observational epidemiology? Int J Epidemiol 2004;33:464-467.

17 Cloos JM, Bocquet V, Rolland-Portal I, Koch P, Chouinard G: Hypnotics and triazolobenzodiazepines - best predictors of high-dose benzodiazepine use: results from the Luxembourg National Health Insurance Registry. Psychother Psychosom 2015;84:273-283.
18 Olfson M, King M, Schoenbaum M: Benzodiazepine use in the United States. JAMA Psychiatry 2015;72:136-142.

19 Chouinard G: Issues in the clinical use of benzodiazepines: potency, withdrawal, and rebound. J Clin Psychiatry 2004;65:7-12.

20 Ciraulo A, Barnhill JG, Greenblatt DJ, Shader RI, Ciraulo AM, Tarmey MF, Molloy MA, Foti ME: Abuse liability and pharmacokinetics of alprazolam in alcoholic men. J Clin Psychiatry 1988;49:333-337.

21 Schmauss C, Apelt S, Emrich HM: The seeking and liking potentials of alprazolam. Am J Psychiatry $1988 ; 145: 128$

22 Teboul E, Chouinard G: A guide to benzodiazepine selection. Part I. Pharmacological aspects. Can J Psychiatry 1990;35:700-710.

23 Teboul E, Chouinard G: A guide to benzodiazepine selection. Part II. Clinical aspects. Can J Psychiatry 1991;36:62-73.

24 Guidi J, Fava GA: Emerging trends in clinical psychology. Riv Psichiatr 2014;49:227. 